\title{
Effects of naturally-arising HIV Nef mutations on cytotoxic T lymphocyte recognition and Nef's functionality in primary macrophages
}

\author{
Philip Mwimanzi, Zafrul Hasan, Ranya Hassan, Shinya Suzu, Masafumi Takiguchi and Takamasa Ueno*
}

\begin{abstract}
Background: Although HIV can infect several cellular subsets, such as $C D 4^{+} T$ lymphocytes and macrophages, it remains unclear whether an HIV infection in macrophages supports cytotoxic T lymphocyte (CTL) escape. Here, we tested two naturally-arising mutations located in the well-conserved polyproline region of Nef for their effects on $C T L$ recognition, Nef's functionality, and viral replication capacity in macrophages. These mutations were selected because they are known to cause CTL escape in the context of T lymphocytes.

Findings: Monocyte-derived macrophages (MDMs) infected with the wild-type virus, but not with variant viruses, were efficiently killed by CTL clones targeting Nef epitopes, VY8 (VPLRPMTY) and RY11 (RPQVPLRPMTY). The CTLescape mutation, $\mathrm{Arg}^{75} \mathrm{Thr}$, or $\mathrm{Arg}^{75} \mathrm{Thr} / \mathrm{Tyr} \mathrm{r}^{85} \mathrm{Phe}$ double mutation, reduced the HLA class I down-regulation activity and, interestingly, increased the susceptibility of virus-infected MDMs to recognition by CTLs targeting a different epitope. The same mutations reduced the CCR5, but not CD4, down-regulation activity. Moreover, the Nef variants were impaired for Hck activation and enhancement of viral replication in MDMs.
\end{abstract}

Conclusions: These results suggest that HIV-infected MDMs are killed by CTLs targeting Nef epitopes, contributing to selection and adaptation of CTL-escape viral variants.

\section{Findings}

Several different cellular subsets such as $\mathrm{CD} 4^{+} \mathrm{T}$ lymphocytes, macrophages, and dendritic cells can be targets for an HIV infection; although they differentially support HIV replication and persistence in vivo [1-3]. Macrophages may be the early target of HIV, but are highly resistant to the cytopathic effects of an HIV infection and continuously produce infectious virions for a long period of time $[4,5]$. It is thought that the differences in fitness of viral replication among the different cellular environments could influence the selection and adaptation of viral quasispecies in these cells. The HLA class I-restricted $\mathrm{CD}^{+}$cytotoxic $\mathrm{T}$ lymphocyte (CTL) response is thought to play an important role in controlling HIV replication [6-8] and to mediate a major selective force for the emergence of viral variants $[9,10]$. Certain CTL escape mutations, in well-conserved regions of Gag and Nef, have been reported to impose

\footnotetext{
* Correspondence: uenotaka@kumamoto-u.ac.jp

Center for AIDS Research, Kumamoto University, Kumamoto, Japan
}

functional constraints on these proteins and to modulate viral replication in the context of $\mathrm{T}$ lymphocytes [11-13]. However, in the context of macrophages, the selection of CTL escape variants and functional adaptation of viral proteins are not yet fully understood. We previously showed that the HLA-B35-restricted CTL responses toward a well-conserved proline-rich region in Nef results in the emergence of a CTL escape mutation, either Arg75Thr or Tyr85Phe, from phylogenetically different viral quasispecies even within an HIVinfected host [13]. These mutations constrain some of the important Nef functions in $\mathrm{CD} 4^{+} \mathrm{T}$ cells [13]. Here we tested whether an HIV-1 infection in macrophages would have any influence on CTL recognition and escape as well as Nef's functionality and adaptation in the infected macrophages.

\section{Susceptibility of HIV-infected macrophages to recognition} by the cognate CTLS

We previously reported that in HIV-infected patients with HLA-B35, the Nef protein elicits dominant CD8 T

\section{() Biomed Central}


cell responses [14], with the short epitope VY8 $\left(\mathrm{Nef}_{78-85}\right.$; VPLRPMTY) being the early epitope, which subsequently shifts to the amino terminal-extended longer epitope RY11 ( $\mathrm{Nef}_{75-85}$; RPQVPLRPMTY) [13]. Autologous virus sequence analysis revealed that the mutations Tyr85 to Phe (85F) and Arg75 to Thr (75T) are associated with the early and chronic phase of an HIV infection, respectively, in HIV-infected individuals with $H L A$ $B 35$ but that these $85 \mathrm{~F}$ and $75 \mathrm{~T}$ mutations are derived from phylogenetically different lineages [13].

We first examined CTL activity toward macrophages infected with HIV-1 strain JRFL, in which nef gene had been replaced with that of strain SF2 (referred as JRFLSF2nef) and its variants. In this JRFL-SF2nef, we had created unique restriction sites, Cla I and Not I adjacent to the ends of the nef open reading frame [15] and confirmed that the resultant viruses, prepared by transfecting $293 \mathrm{~T}$ cells with JRFL and JRFL-SF2nef, had comparable replication capacity in primary monocytederived macrophages (MDMs) (data not shown). To prepare mature MDMs, $\mathrm{CD} 14^{+}$cells were isolated from PBMCs of HIV-negative donors, in accordance with the human experimentation guidelines of Kumamoto University, and cultured for 7 days in the presence of 100 $\mathrm{ng} / \mathrm{ml}$ of macrophage colony-stimulating factor (Peprotech GmbH, Germany). Previously established CTLs, specific for VY8 and RY11, [13,14] were highly cytotoxic toward MDMs infected with wild-type (wt) HIV-1, suggesting that HIV-infected MDMs were a preferable target for CTLs. The VY8-specific CTLs showed higher cytotoxicity toward wt virus-infected MDMs than did the RY11-specific CTLs (Figure 1), in good agreement with the observation obtained with HIV-infected CD4 ${ }^{+}$ $\mathrm{T}$ cells $[13,16]$. In contrast, VY8- and RY11-specific CTLs failed to kill primary MDMs infected with $85 \mathrm{~F}$ and $75 \mathrm{~T}$ viruses, respectively (Figure 1 ), indicating that the $85 \mathrm{~F}$ and $75 \mathrm{~T}$ single mutations conferred escape from CTLs specific for VY8 and RY11, respectively, but not simultaneously. In contrast, the TF virus could escape from both types of CTLs (Figure 1). It should be noted that Western blot analysis of Nef proteins in virus-producing cells showed a comparable level of Nef expression among wt and all variant viruses except for $\Delta$ Nef (data not shown).

\section{Effects of the Nef mutations on Hck activation}

Nef is known to associate via its PxxP motif with the SH3 domain of several different cellular kinases including Hck $[17,18]$. We tested whether the CTL-escape variants in the PxxP region would affect the Hck activation by Nef by using the in vitro Hck activation assay as described earlier [19] (Figure 2A). Expectedly, the wildtype Nef showed robust Hck activation; whereas the AxxA variant Nef (Pro76Ala and Pro79Ala) did not show substantial activation (Figure $2 \mathrm{~B}$ ). The $85 \mathrm{~F}$ variant Nef did not affect Hck activation, whereas the Hck activation was substantially reduced by the $75 \mathrm{~T}$ and $\mathrm{TF}$ variants of Nef (Figure 2B). These results suggest that CTL-escape variants in the PxxP motif affect Hck activation in macrophages.

\section{Effects of the Nef mutations on HLA class I down- regulation}

Because Nef helps HIV-infected cells to evade CTL lysis by down-modulating cell-surface HLA-I and the PxxP motif is critical for this activity $[13,20,21]$, we examined the HLA-I down-regulation activity by Nef in MDMs infected with wt and variant viruses by flow cytometry (Figure 3A). The surface levels of HLA-I within p24 ${ }^{+}$ subsets in wt virus-infected MDMs were much reduced compared with those in uninfected cells (Figure 3B) and that no HLA-I down-regulation was observed in $\Delta \mathrm{Nef}$ virus-infected MDMs (Figure 3B). In contrast, both the $75 \mathrm{~T}$ and the TF variant viruses showed substantially diminished down-regulation activity; whereas the $85 \mathrm{~F}$ variant virus showed down-regulation activity comparable to that of the wt (Figure 3B).

\section{Susceptibility of HIV-infected MDMs to recognition by CTLs of another specificity}

We postulated that the impaired Nef-mediated downregulation activity of HLA-I in MDMs could influence the susceptibility to killing of HIV-infected MDMs by CTLs. To test this, we first created the variant virus having M20A or P82A (numbering based on the SF2 strain) because these mutations have been shown to completely disrupt the Nef-mediated HLA-I down-regulation activity $[22,23]$. We then assessed the cytolytic activity of CTL clones specific for another Nef epitope presented by HLA-A24 ( Nef $_{138-147}$ : RYPLTFGWCF) toward MDMs infected with wt, M20A, or P82A viruses. Although the amino-acid sequences in the epitope region of A24-Nef were the same among the wt and these variant viruses tested, the CTL-mediated killing activity toward MDMs infected with M20A and P82A variant viruses was much increased compared to those infected with the wt virus (Figure 4).

Next, we also determined CTL cytotoxic activity toward MDMs infected with 75T, 85F, and TF variant viruses. The A24-Nef CTLs showed the most potent activity toward MDMs infected with either the 75T or TF variant viruses; whereas their cytotoxic activity was less potent toward MDMs infected with either the wt or the 85F mutant virus (Figure 4). These data suggest that the diminished HLA-I down-regulation (i.e., increased level of cell-surface HLA-I) in MDMs infected with the $75 \mathrm{~T}$ and the TF mutant viruses (Figure 3) resulted in increased susceptibility to killing by CTLs of another specificity (Figure 4), leading to a possible 


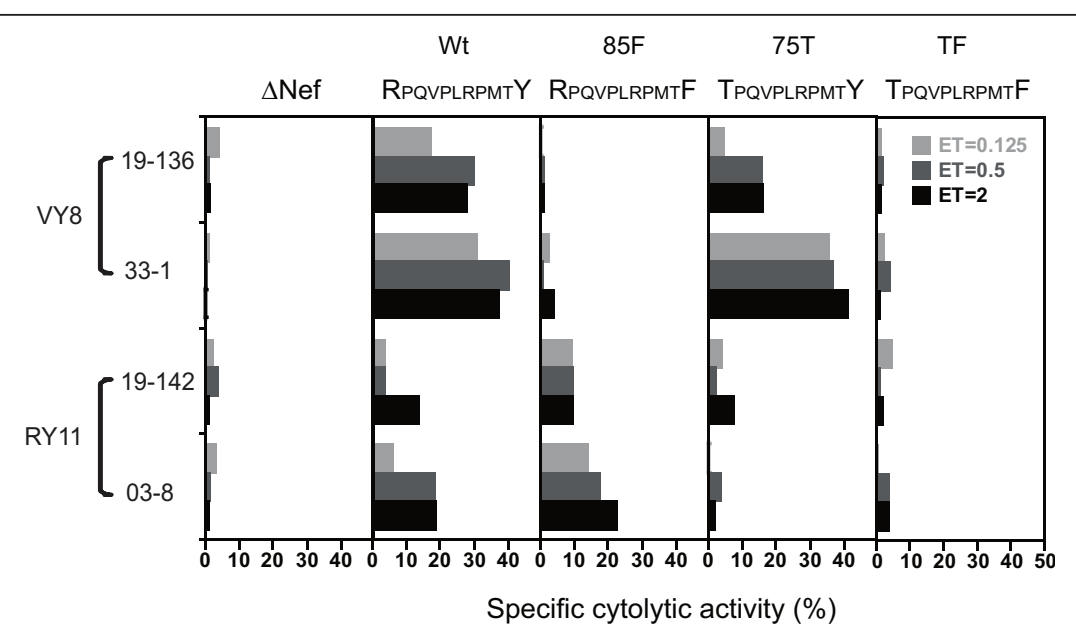

Figure 1 Susceptibility of HIV-infected MDMs to recognition by the cognate CTLs. Cytotoxic activity of HLA-B35-restricted CTL clones specific for VY8 (VPLRPMTY) and RY11 (RPQVPLRPMTY) epitopes in Nef toward HIV-infected MDMs is shown. MDMs were isolated from an HIVnegative donor $\left(H L A-B^{*} 35: 01^{+}\right)$and infected with wild-type or one of the variant viruses indicated. The resultant HIV-infected MDMs $\left(2 \times 10^{3} /\right.$ well) were then mixed with $C T L$ clones at various effector-to-target cell ratios $(E / T)$ for $6 \mathrm{hr}$ at $37^{\circ} \mathrm{C}$ after having been labeled with ${ }^{51} \mathrm{Cr}$. The frequency of HIV-infected cells among target cells as determined by intracellular p24 Ag expression was 48.7, 55.4, 51.0, and 48.8\% for wt, 85F, 75T, and TF variants, respectively. CTL 19-136 and 19-142 were derived from the same HIV-infected donor (019), and CTL 33-1 and 03-8 were derived from different donors, 033 and 03, respectively. CTL activity toward uninfected cells was deducted from the data as background. An additional experiment showed similar results.

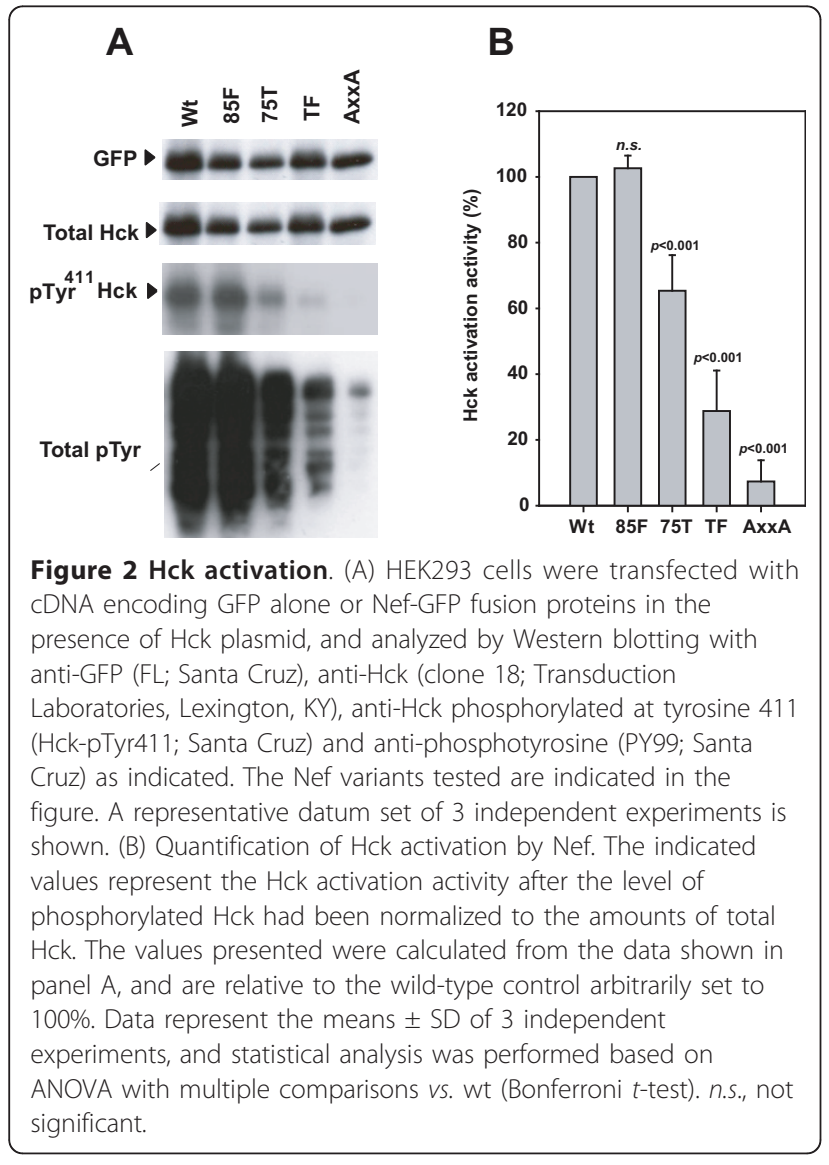

selective disadvantage for the variant viruses under antiHIV CTL responses.

\section{Effects of the Nef mutations on down-regulation of viral receptors}

We also examined whether Nef's down-regulation activity of viral receptors, i.e., CD4 and CCR5, could be influenced by the mutations in HIV-infected MDMs (Figure 5A). The cell-surface expression of CCR5 was substantially reduced in wt virus-infected MDMs but not affected in the $\Delta \mathrm{Nef}$ variant virus-infected ones (Figure $5 \mathrm{~B}$ ). Interestingly, the $85 \mathrm{~F}$ variant virus showed CCR5 down-regulation activity comparable to that of the wt virus; whereas the $75 \mathrm{~T}$ and $\mathrm{TF}$ variant were substantially impaired in this activity in MDMs (Figure 5B). In contrast, $\mathrm{CD} 4$ down-regulation activity was not affected for all of the viruses with mutated Nefs except for $\Delta \mathrm{Nef}$ (Figure $5 \mathrm{C}$ ), consistent with the observation that CD4 down-regulation activity is mediated by a specific region in Nef other than the PxxP motif [21].

\section{Effects of the Nef mutations on viral replication}

We finally examined whether the mutations would differently affect the enhancement of viral replication in MDMs. In MDMs from $2 \mathrm{HIV-negative} \mathrm{donors,} \mathrm{the} \mathrm{wt}$ HIV-1 showed the highest replication among the various viruses tested; whereas the $\Delta \mathrm{Nef}$ variant showed much decreased replication (Figure 6A), consistent with the 


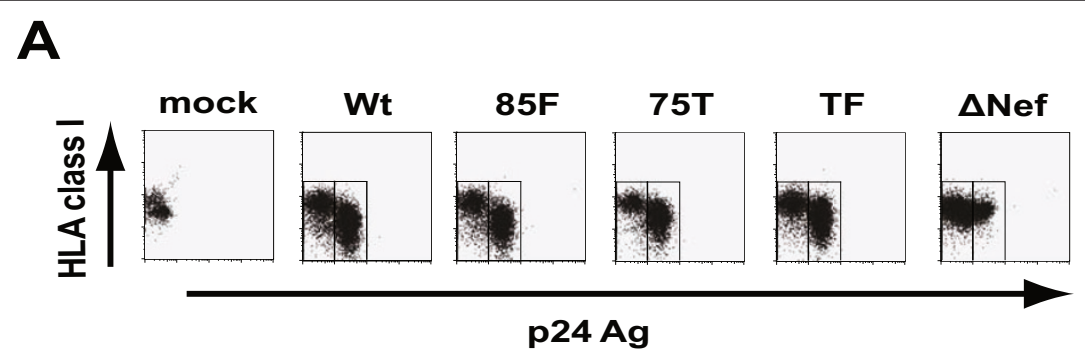

B

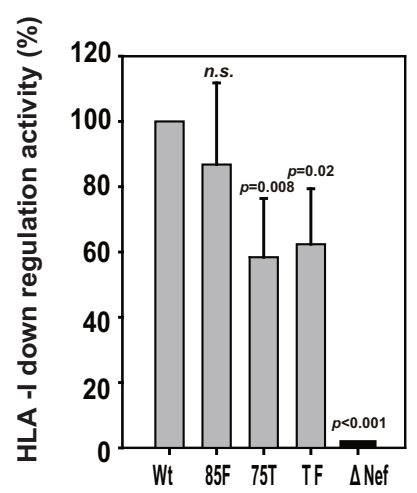

Figure 3 HLA class I down-regulation in HIV-infected MDMs. (A) MDMs prepared from an HIV-negative donor were infected with wild-type or variant viruses as indicated. Cells were stained with 7-amino-actinomycin D (7-AAD; BD Biosciences, CA) and anti-HLA class I allotype antibody SFR8-B6 followed by intracellular staining with FITC-labeled anti-p24 Gag mAb (KC-57; Beckman Coulter, CA) as described before [13]. In flow cytometric analysis (FACS Canto II, BD Biosciences), cells negative for 7-AAD were gated and analyzed for their fluorescence intensity for HLA class I and p24 Gag. (B) The same experiment as above was done by using 3 additional HIV-negative donors. The HLA class I allotype-specific antibodies used were either SFR8-B6 or A11,1 M as appropriate for each donor. The relative down-regulation activity of HLA class I by wt Nef and its variants is presented relative to that of the wild-type Nef activity set to $100 \%$. Data represent the means \pm SD of all 4 donors, and statistical analysis was performed based on ANOVA with multiple comparisons vs. wt (Bonferroni $t$-test). n.s., not significant.

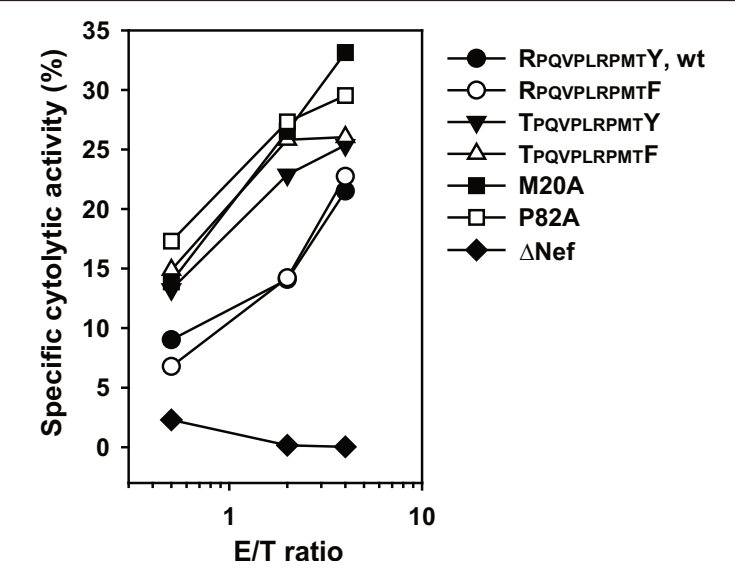

Figure 4 Susceptibility of HIV-infected MDMs to recognition by CTLs of another specificity. MDMs prepared from an HIV-negative donor $\left(H L A-A^{*} 24: 02^{+}\right)$infected with the indicated viruses were used as target cells for cytolysis by an HLA-A24-restricted CTL clone specific for the Nef epitope ( Nef $_{138-147}$ : RYPLTFGWCF). The frequency of HIV-infected cells among the target cells, as determined by intracellular p24 Ag expression, was 41.4, 48.3, 44.5, 40.8, 40.0, and 45.0\% for wt, 85F, 75T, TF, M20A, and P82A variants, respectively. CTL activity toward uninfected cells was deducted from the data as background. An additional experiment showed similar results. previous observation [24]. The replication of the $85 \mathrm{~F}$ variant virus was partially impaired in MDMs from one of the donors and was comparable to that of the wt virus in MDMs from the other donor (Figure 6A). In contrast, the replication of the $75 \mathrm{~T}$ and TF variant viruses was impaired in MDMs from both donors (Figure 6A). To account for this donor variability, we summarized the results from a total of 5 donors in Figure 6B. Because the peak of the virus replication was between 6 to 12 days after infection, depending on the donor and the virus, the peak p24 Ag values of each of the viruses are presented and were used for statistical analysis (Figure 6B). The $75 \mathrm{~T}$ and the TF variant viruses showed significantly diminished capacity for viral replication compared with the wt; whereas the $85 \mathrm{~F}$ virus did not show much difference in replication capacity (Figure 6B).

\section{Discussion and Conclusions}

Although the Nef protein is thought to have very high mutational plasticity, we showed here that the naturally-arising CTL escape variants in the wellconserved PxxP region in Nef alone or in combination can modulate some pathogenic functions of Nef in the 


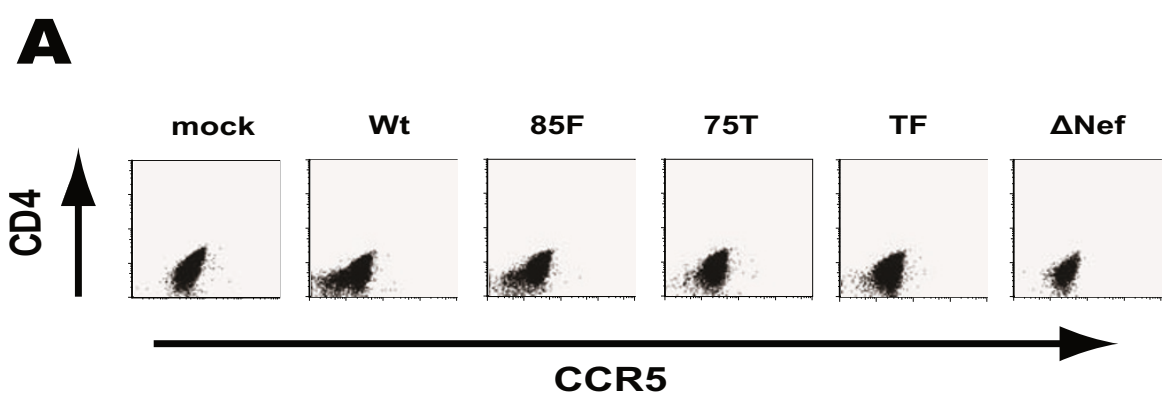

$\mathbf{B}$

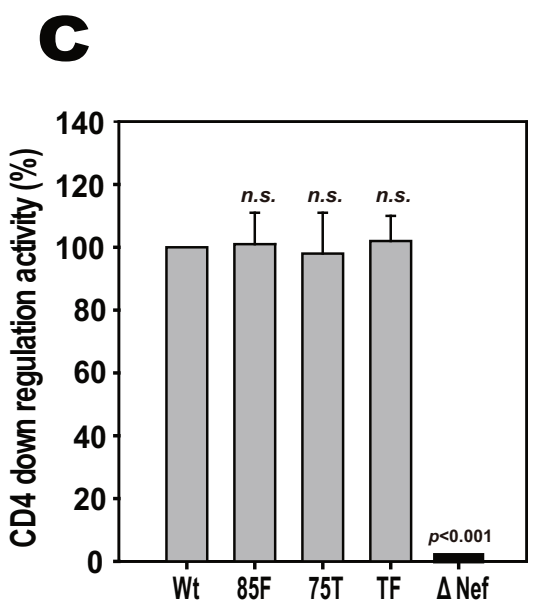

Figure 5 Viral receptor down-regulation in HIV-infected MDMs. (A) MDMs prepared from an HIV-negative donor were infected with wt or variant viruses as indicated. The cells were stained with 7-AAD and allophycocyanin-Cy7 anti-human CD4 mAb (Biolegend, CA) and phycoerythrin-Cy7-conjugated anti-human CCR5 mAb (BD Biosciences) followed by intracellular staining with antibody against p24 Gag. In flow cytometric analysis, cells negative for 7-AAD and positive for p24 Gag were gated and analyzed for their fluorescence intensity for CD4 and CCR5. (B, C) The same experiment as above was done by using 3 additional HIV-negative donors. The relative down-regulation activity of wt Nef and its various variants toward CCR5 (panel B) and CD4 (panel C) is presented, with the wt Nef activity set to 100\%. Data represent the means \pm SD of all 4 donors, and statistical analysis was performed based on ANOVA with multiple comparisons vs. wt (Bonferroni t-test). n.s., not significant.

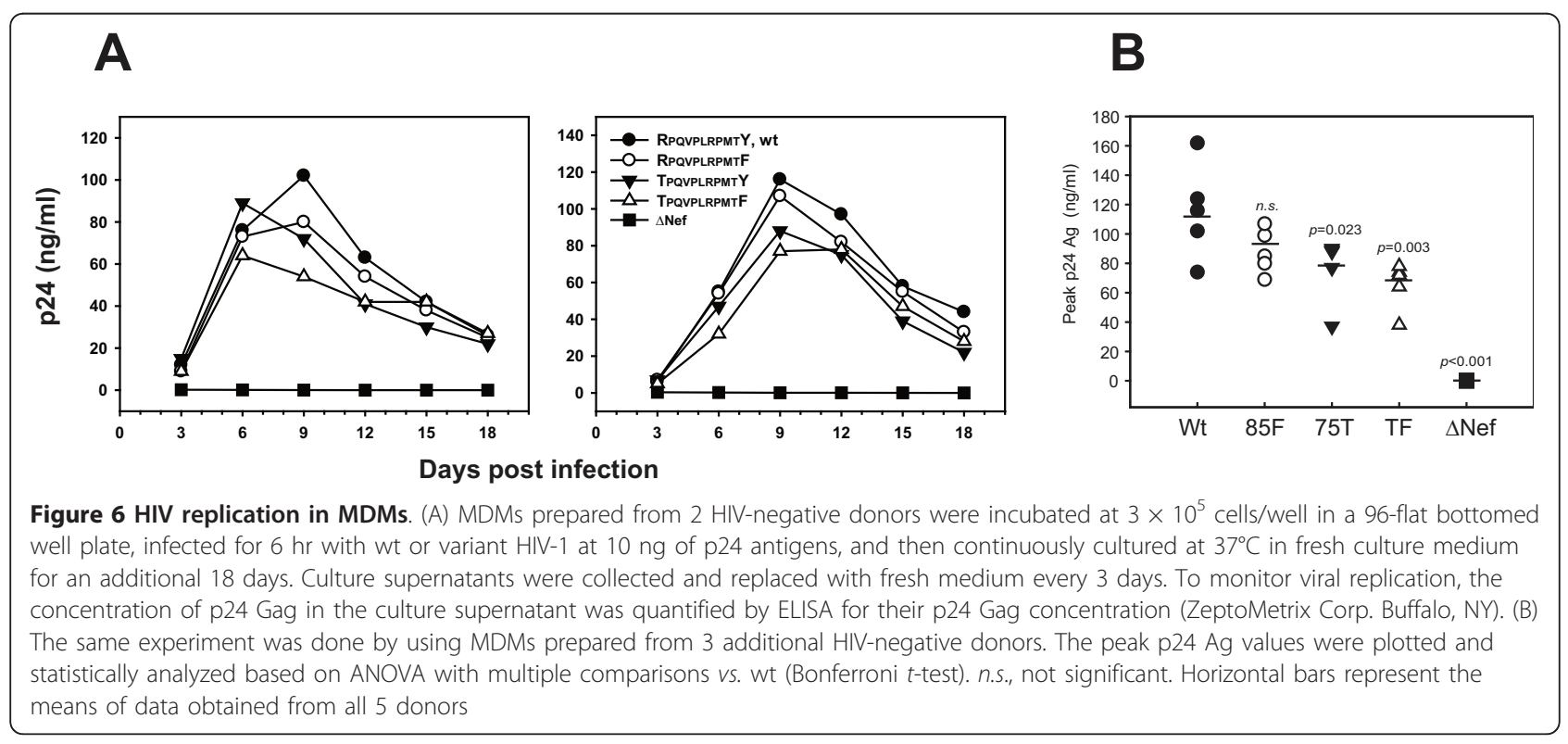


context of human primary macrophages infected with a CCR5-tropic virus. There are 2 different aspects of CTLmediated functional constraints on the PxxP-dependent Nef activities in MDMs reported here, one through immune evasion activity (HLA-I down-regulation activity) and the other acting on the intrinsic capacity to boost viral replication and persistence (Hck activation, viral co-receptor down-regulation activity, and enhancement of viral replication). In particular, one of the single mutants, 75T, impaired these Nef activities in MDMs infected with a CCR5-tropic virus. This is in line with the previous report showing that $75 \mathrm{~T}$ mutation modulated Nef-stimulated viral replication in immature dendritic cell/T cell cocultures infected with a CCR5-tropic virus [25] although this mutation alone had virtually no influence on the same Nef activities in primary $\mathrm{CD}_{4}^{+} \mathrm{T}$ cells infected with a CXCR4tropic virus in the previous study [13]. In addition, the 75T mutation, located outside the VY8 epitope, reduced the cytolytic activity of VY8-specific CTLs in the context of $\mathrm{CD}^{+} \mathrm{T}$ cells [13], but did not affect their cytolytic activity in the context of MDMs (Figure 1), suggesting the differential intracellular processing of the VY8 peptide between $\mathrm{CD}^{+} \mathrm{T}$ cells and MDMs. This observation is in line with the previous report showing a substantial difference in intracellular processing of antigenic HIV peptides between monocytes and lymphocytes [26]. Overall, these results suggest that an antigenic variation of viruses can differentially influence viral replication and persistence between cellular subsets because of their different effects on the intracellular antigen-processing machinery, the susceptibility to CTL killing, as well as the fitness cost to viral replication.

Of particular interest are the data showing that the CTL-escape Nef mutation, 75T, impaired HLA-I downregulation activity by Nef and rendered the HIV-infected MDMs more susceptible to killing by CTLs with another specificity. Such phenomenon was also observed in the context of CD4 $4^{+} \mathrm{T}$ cells in our previous study [13]. However, wt-virus-infected cells, regardless of CD4 ${ }^{+} \mathrm{T}$ cells or MDMs, could be killed to some extent by CTLs, suggesting that the Nef-mediated HLA-I downregulation is insufficient for HIV to escape from CTL recognition and that, CTL-escape variant viruses are selected and emerged. Conversely, Swigut et al., [27] reported that monkeys infected with SIV containing nef mutations that selectively eliminated $\mathrm{MHC}$ down-regulation activity exhibited higher level of SIV-specific CD8 T cell responses. In any event, an important question remains to be addressed which is how significant is Nefmediated HLA-I down-regulation activity for HIV replication and persistence in HIV-infected humans.

Although HLA-B*35-restricted CTLs targeting PxxP region of Nef can impose functional constraints in viral replication in this study, we did not find any beneficial effects on clinical parameters (such as CD4 count and viral load) in HIV-infected patients with HLA-B*35 as well as those with HLA-B*35 and HLA-A*24 in our cohort to date (data not shown). Functional impairment in Nef induced by CTL-escape variants could be compensated later by mutations at secondary sites in Nef. For example, an inverse dose-response relationship has been observed between the number of CTL-escape mutations in Nef and CD4 counts in patients in a large population study [28]. Therefore, only some CTL-escape variants may play a role in modulating Nef functions in vivo, such as in the case of HLA-B57 $7^{+}$elite suppressors [29]. Further studies using a large number of clinicallyisolated nef alleles are needed to extend this observation, such as how Nef-specific CTL responses, Nef functions, and clinical outcome of HIV-infected individuals are related to each other at the population level.

\section{Acknowledgements}

We thank Dr. M Fujiwara and Ms. S. Doki for their great help. This research was supported by a grant-in-aid for scientific research from the Ministry of Education, Science, Sports, and Culture of Japan and by a grant-in-aid for AIDS research from the Ministry of Health, Labor, and Welfare of Japan.

\section{Authors' contributions}

PM, MT, and TU designed the study. PM, ZH, RH, SS, and TU conducted the experiments. PM, SS, and TU wrote the paper. All authors read and approved the final manuscript.

\section{Competing interests}

The authors declare that they have no competing interests.

Received: 11 March 2011 Accepted: 22 June 2011

Published: 22 June 2011

\section{References}

1. Eckstein DA, Penn ML, Korin YD, Scripture-Adams DD, Zack JA, Kreisberg JF, Roederer M, Sherman MP, Chin PS, Goldsmith MA: HIV-1 actively replicates in naive CD4+ T cells residing within human lymphoid tissues. Immunity 2001, 15:671-682.

2. Keele BF, Tazi L, Gartner S, Liu Y, Burgon TB, Estes JD, Thacker TC, Crandall KA, McArthur JC, Burton GF: Characterization of the follicular dendritic cell reservoir of human immunodeficiency virus type 1. J Virol 2008, 82:5548-5561.

3. Zhu T, Muthui D, Holte S, Nickle D, Feng F, Brodie S, Hwangbo Y, Mullins I, Corey L: Evidence for human immunodeficiency virus type 1 replication in vivo in CD14+ monocytes and its potential role as a source of virus in patients on highly active antiretroviral therapy. J Virol 2002, 76:707-716.

4. Aquaro S, Bagnarelli P, Guenci T, De Luca A, Clementi M, Balestra E, Caliò R, Perno CF: Long-term survival and virus production in human primary macrophages infected by human immunodeficiency virus. J Med Virol 2002, 68:479-488.

5. Brown A, Zhang H, Lopez P, Pardo CA, Gartner S: In vitro modeling of the HIV-macrophage reservoir. J Leu Biol 2006, 80:1127-1135.

6. Borrow PH, Lewicki BH, Hahn GM, Shaw MB, Oldstone : Virus-specific CD8 ${ }^{+}$ cytotoxic T-lymphocyte activity associated with control of viremia in primary human immunodeficiency virus type 1 infection. J Virol 1994, 68:6103-6110.

7. Koup RA, Safrit JT, Cao Y, Andrews CA, McLeod G, Borkowsky W, Farthing C, Ho DD: Temporal association of cellular immune responses with the initial control of viremia in primary human immunodeficiency virus type 1 syndrome. J Virol 1994, 68:4650-4655.

8. Ogg GS, Jin X, Bonhoeffer S, Dunbar PR, Nowak MA, Monard S, Segal JP, Cao Y, Rowland-Jones SL, Cerundolo V, et al: Quantitation of HIV-1-specific 
cytotoxic T lymphocytes and plasma load of viral RNA. Science 1998, 279:2103.

9. Goulder PJR, Watkins DI: HIV and SIV CTL escape: implications for vaccine design. Nat Rev Immunol 2004, 4:630-640.

10. Motozono C, Mwimanzi P, Ueno T: Dynamic interplay between viral adaptation and immune recognition during HIV-1 infection. Protein \& Cell 2010, 1:514-519.

11. Brockman MA, Schneidewind A, Lahaie M, Schmidt A, Miura T, DeSouza I, Ryvkin $F$, Derdeyn CA, Allen $S$, Hunter $E$, et al: Escape and compensation from early HLA-B57-mediated cytotoxic T-lymphocyte pressure on human immunodeficiency virus type $1 \mathrm{Gag}$ alter capsid interactions with cyclophilin A. J Virol 2007, 81:12608-12618.

12. Schneidewind A, Brockman MA, Sidney J, Wang YE, Chen H, Suscovich TJ, Li B, Adam Rl, Allgaier RL, Mothe BR, et al: Structural and functional constraints limit options for cytotoxic T-lymphocyte escape in the immunodominant HLA-B27-restricted epitope in human immunodeficiency virus type 1 capsid. J Virol 2008, 82:5594-5605.

13. Ueno T, Motozono C, Dohki S, Mwimanzi P, Rauch S, Fackler OT, Oka S, Takiguchi M: CTL-mediated selective pressure influences dynamic evolution and pathogenic functions of HIV-1 Nef. J Immunol 2008 180:1107-1116.

14. Ueno T, Idegami Y, Motozono C, Oka S, Takiguchi M: Altering effects of antigenic variations in HIV-1 on antiviral effectiveness of HIV-specific CTLs. J Immunol 2007, 178:5513-5523.

15. Fujiwara M, Takiguchi M: HIV-1-specific CTLs effectively suppress replication of HIV-1 in HIV-1-infected macrophages. Blood 2007, 109:4832-4838.

16. Motozono C, Yanaka S, Tsumoto K, Takiguchi M, Ueno T: Impact of intrinsic cooperative thermodynamics of peptide-MHC complexes on antiviral activity of HIV-specific CTL. J Immunol 2009, 182:5528-5536.

17. Briggs SD, Sharkey M, Stevenson M, Smithgall TE: SH3-mediated Hck tyrosine kinase activation and fibroblast transformation by the Nef protein of HIV-1. J Biol Chem 1997, 272:17899-17902.

18. Trible RP, Emert-Sedlak L, Smithgall TE: HIV-1 Nef selectively activates Src family kinases Hck, Lyn, and c-Src through direct SH3 domain interaction. J Biol Chem 2006, 281:27029-27038.

19. Hassan R, Suzu S, Hiyoshi M, Takahashi-Makise N, Ueno T, Agatsuma T, Akari H, Komano J, Takebe Y, Motoyoshi K, Okada S: Dys-regulated activation of a Src tyrosine kinase Hck at the Golgi disturbs Nglycosylation of a cytokine receptor Fms. J Cell Physiol 2009, 221:458-468.

20. Collins KL, Chen BK, Kalams SA, Walker BD, Baltimore D: HIV-1 Nef protein protects infected primary cells against killing by cytotoxic $T$ lymphocytes. Nature 1998, 391:397-401.

21. Saksela K, Cheng G, Baltimore D: Proline-rich (PxxP) motifs in HIV-1 Nef bind to $\mathrm{SH} 3$ domains of a subset of Src kinases and are required for the enhanced growth of Nef+ viruses but not for down-regulation of CD4. Embo J 1995, 14:484-491

22. Akari H, Arold S, Fukumori T, Okazaki T, Strebel K, Adachi A: Nef-induced major histocompatibility complex class I down-regulation is functionally dissociated from its virion incorporation, enhancement of viral infectivity, and CD4 down-regulation. J Virol 2000, 74:2907-2912.

23. Yamada T, Kaji N, Odawara T, Chiba J, Iwamoto A, Kitamura Y: Proline 78 is crucial for human immunodeficiency virus type 1 Nef to down-regulate class I human leukocyte antigen. J Virol 2003, 77:1589-1594.

24. Miller MD, Warmerdam MT, Gaston I, Greene WC, Feinberg MB: The human immunodeficiency virus-1 nef gene product: a positive factor for viral infection and replication in primary lymphocytes and macrophages. $J$ Exp Med 1994, 179:101-113.

25. Fackler OT, Wolf D, Weber HO, Laffert B, D'Aloja P, Schuler-Thurner B, Geffin R, Saksela K, Geyer M, Peterlin BM: A natural variability in the proline-rich motif of Nef modulates HIV-1 replication in primary T cells. Current Biology 2001, 11:1294-1299.

26. Lazaro E, Godfrey SB, Stamegna P, Ogbechie T, Kerrigan C, Zhang M, Walker BD, Le Gall S: Differential HIV epitope processing in monocytes and CD4 T cells affects cytotoxic T lymphocyte recognition. J Infect Dis 2009, 200:236-243.

27. Swigut T, Alexander L, Morgan J, Lifson J, Mansfield KG, Lang S, Johnson RP, Skowronski J, Desrosiers R: Impact of Nef-mediated downregulation of major histocompatibility complex class I on immune response to simian immunodeficiency virus. J Virol 2004, 78:13335-13344.
28. Brumme ZL, Brumme CJ, Heckerman D, Korber BT, Daniels M, Carlson J, Kadie C, Bhattacharya T, Chui C, Szinger J, et al: Evidence of differential HLA class I-mediated viral evolution in functional and accessory/ regulatory genes of HIV-1. PLoS Pathogens 2007, 3:e94.

29. Bailey JR, Brennan TP, O'Connell KA, Siliciano RF, Blankson JN: Evidence of CD8+ T-cell-mediated selective pressure on human immunodeficiency virus type 1 nef in HLA-B*57+ elite suppressors. J Virol 2009, 83:88-97.

doi:10.1186/1742-4690-8-50

Cite this article as: Mwimanzi et al.: Effects of naturally-arising HIV Nef mutations on cytotoxic T lymphocyte recognition and Nef's functionality in primary macrophages. Retrovirology 2011 8:50

\section{Submit your next manuscript to BioMed Central and take full advantage of:}

- Convenient online submission

- Thorough peer review

- No space constraints or color figure charges

- Immediate publication on acceptance

- Inclusion in PubMed, CAS, Scopus and Google Scholar

- Research which is freely available for redistribution 